\title{
Local Dynamics of cis-1,4-Polybutadiene and cis-1,4-Polyisoprene. A Comparative Study Based on Cooperative Kinematics Theory and NMR Experiments
}

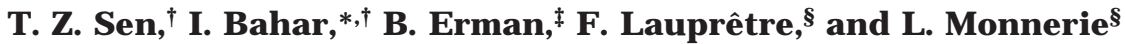 \\ Chemical Engineering Department, Polymer Research Center, Bogazici University, and TUBITAK \\ Advanced Polymeric Materials Research Center, Bebek 80815, I stanbul, Turkey, Faculty of \\ Engineering and Natural Sciences, Sabanci University, Bankalar Cad No. 2, Karakoy 80020, \\ I stanbul, Turkey, and Ecole Supérieure de Physique et Chimie Industrielles, PCSM, 10 rue Vauquelin, \\ 75231 Paris Cedex 05, France
}

Received J uly 15, 1998; Revised Manuscript Received J anuary 15, 1999

\begin{abstract}
Cooperative motions of cis-1,4-polybutadiene (cis-PB) and cis-1,4-polyisoprene (cis-PI) in the bulk state are analyzed and compared using the cooperative kinematics (CK) theory. The mechanisms of relaxation on a local scale are closely similar for the two polymers in dense media, resulting from the same geometry of the backbone, which is the major determinant of the types of accessible cooperative conformational rearrangements. The correlation times for the reorientation of $\mathrm{C}-\mathrm{H}$ bonds of cis-PI are found to be about 1 order of magnitude longer than those for cis-PB at the same effective friction coefficient. The results are in accordance with previous NMR experiments which indicated a ratio of about 10 . The molecular characteristics responsible for this difference in the local mobilities of the two polymers are examined. Three sources are identified: (i) the excess vol ume swept by the moving units in cis-PI due to the $\mathrm{CH}_{3}$ units appended to the backbone, (ii) the higher short-range conformational potential energies in cis-PI compared to those in cis-PB, and (iii) the restriction in the number of accessible isomeric transitions in cis-PI compared to those of cis-PB, which is due to the excluded-volume effect.
\end{abstract}

\section{Introduction}

Local dynamics of polymers in the bulk state occurs through librations of backbone bonds about rotational energy minima and through transitions between rotational isomeric states (RIS). ${ }^{1,2}$ The dynamics is affected mainly by four factors. ${ }^{3}$ The first is backbone geometry. This is an intrinsic structural property of the investigated chain, irrespective of environment. Bond lengths and bond angles affect the extent of the displacement of atoms in the vicinity of the bond undergoing a rotational motion. For most polymers, the distortions of bond lengths and bond angles are opposed by large potentials, such that these are almost fixed in comparison to dihedral angles. The second factor involves the side groups appended to the backbone. The geometry of the side groups and their size lead to differences in the extent of volume swept by the moving atoms during a rotameric transition. The displacements of backbone and side chain atoms during rotameric jumps are also functions of the instantaneous conformation of the chain. The third factor is the environment. Closely related to the factor of backbone and side chain geometry is the extent of friction opposing the motion. This effect is intermolecular in origin and becomes particularly important in dense media. The work done against environment during the conformational motions accompanying the isomeric transition of a given bond increases with the effective frictional resistance. The fourth factor is the short-range conformational energetics of the chain. Precisely, the shape of the energy map

\footnotetext{
* To whom correspondence should be addressed at Bogazici University.

† Bogazici University and TUBITAK Advanced Polymeric Materials Research Center.

₹ Sabanci University.

$\S$ PCSM.
}

near the minima around which the librational motions take place and the heights of the energy barriers between RIS affect the mechanism of relaxation. These energies are expressed in terms of the rotational potentials of bonds. They include the intrinsic rotational preferences of the individual bonds and the first-order interactions between the near-neighboring units along the backbone.

The effect of these factors on the correlation time $\tau_{c}$ associated with local motions is conveniently summarized in the expression ${ }^{4}$

$$
\tau_{\mathrm{c}} \sim \tau_{0}\left(\xi \sum_{\mathrm{i}} \delta \mathbf{R}_{\mathrm{i}} \cdot \delta \mathbf{R}_{\mathrm{i}}\right) \exp (\Delta \mathrm{V} / \mathrm{RT})
$$

based on Kramers' rate theory in the high-friction limit. Here, $\tau_{0}$ is a front factor accounting for the effects of the individual chain geometry-backbone and side chains-on the nature of isomeric jumps, $\xi$ is the effective friction coefficient, $\sum \delta \mathbf{R}_{\mathrm{i}} \cdot \delta \mathbf{R}_{\mathrm{i}}$ is the cumulative square displacements of all atoms, and $\Delta \mathrm{V}$ is the conformational energy change involved in the motion. The above correlation time $\tau_{\mathrm{c}}$ characterizes the time decay of the normalized autocorrelation function (expressed by the second-order Legendre polynomial) associated with the reorientation of the internuclear vector observed in NMR.

Let us now investigate how eq 1 may be utilized for extracting information on relaxation times. First, a chain in a specific original conformation is chosen. The bond of interest is rotated from one isomeric state to another by imposing successive incremental changes in its dihedral angle. The response of the chain to this rotameric transition depends on the local conformation near the rotating bond along the chain. This response may conveniently be determined by the use of the 
cooperative kinematics (CK) theory. ${ }^{3-9}$ According to the CK method, the conformational rearrangements of the chain are subject to the condition of minimization of the total energy change, here comprising the work W done against friction and the overall change in the conformational energy $\Delta \mathrm{V}$. The work done against friction at each incremental step is given by ${ }^{3-9}$

$$
\delta \mathrm{W}=(\xi / \delta \mathrm{t}) \sum_{\mathrm{i}} \delta \mathbf{R}_{\mathrm{i}} \cdot \delta \mathbf{R}_{\mathrm{i}}
$$

where $\delta$ t is the time step during which the incremental change takes place.

There are $n+4$ degrees of freedom in the CK model for the backbone, $\mathrm{n}-2$ internal dihedral angles, and 6 external degrees of freedom (translation and rotation). One of the bonds is assumed to undergo an isomeric transition, and the accompanying change in the remaining $n+3$ degrees of freedom is calculated. Minimization of $\delta \mathrm{W}+\Delta \mathrm{V}$ with respect to these degrees of freedom leads to $n+3$ coupled equations. Their solution yields the optimal atomic displacements $\delta \mathbf{R}_{\mathrm{i}}$ and, thereby, the two quantities of interest, $\sum \delta \mathbf{R}_{\mathrm{i}} \cdot \delta \mathbf{R}_{\mathrm{i}}$ and $\Delta \mathrm{V}$, which may be readily inserted into eq 1 . The explicit form of the $n$ +3 linear equations and their analytical solution are presented in our previous work. ${ }^{5}$

In eq 1 , the front term $\tau_{0}$ is geometric in nature. It describes the time scale involved in the reorientation of a chain-affixed vector during local relaxation. Reorientations in the form of large angle jumps lead to a faster time decay of orientational correlations. Small, diffusive reorientations lead to slower decay. The effective front factors $\tau_{0}$ for angular motions of different step sizes were determined by a Monte Carlo simulation technique, leading to a functional relationship between the size of the jump angles and $\tau_{0}$. The sizes of reorientational jumps for a given bond are evaluated by the $\mathrm{CK}$ method, so as to assess the corresponding $\tau_{0}$ value. ${ }^{4}$

In the present study, we compare the local dynamics of cis-1,4-polybutadiene (cis-PB) and cis-1,4-polyisoprene (cis-PI) at the same effective friction coefficient. The importance of comparing the characteristic relaxation times of bulk polymers at the same friction coefficient has been discussed before. ${ }^{10}$ The local dynamics of these two systems has been studied in much detail, both by experimental means ${ }^{10-13}$ and by simulations. ${ }^{14-22}$ Results of CK calculations for cis-PB were presented in our earlier work.4,7,8 Results for cis-PI presented in the present paper are new. The comparison shows that the mechanisms of motion of the backbone are approximately identical for the two systems, apart from a slight overall increase in the sizes of the atomic displacements required to accommodate a given bond rotation in cis$\mathrm{PI}$ compared to those in cis-PB. The existence of a methyl side group in the repeat units of cis-PI causes, however, a significant increase in the cumulative square distance $\sum \delta \mathbf{R}_{\mathrm{i}} \cdot \delta \mathbf{R}_{\mathrm{i}}$ traveled by all atoms (including side groups) during conformational changes. More work is therefore required for completing an isomeric transition in cis-PI compared to that for the isomerization of cisPB bonds; and this difference is directly manifested as an increase in the correlation times observed by ${ }^{13} \mathrm{C}$ NMR.

\section{Model and Method}

The CK theory and its application to various polymers together with the comparison with results from molec-

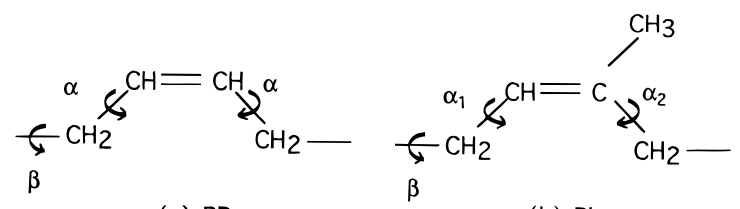

(a) PB

(b) $\mathrm{PI}$

Figure 1. Repeat units of (a) cis-PB and (b) cis-PI chains.

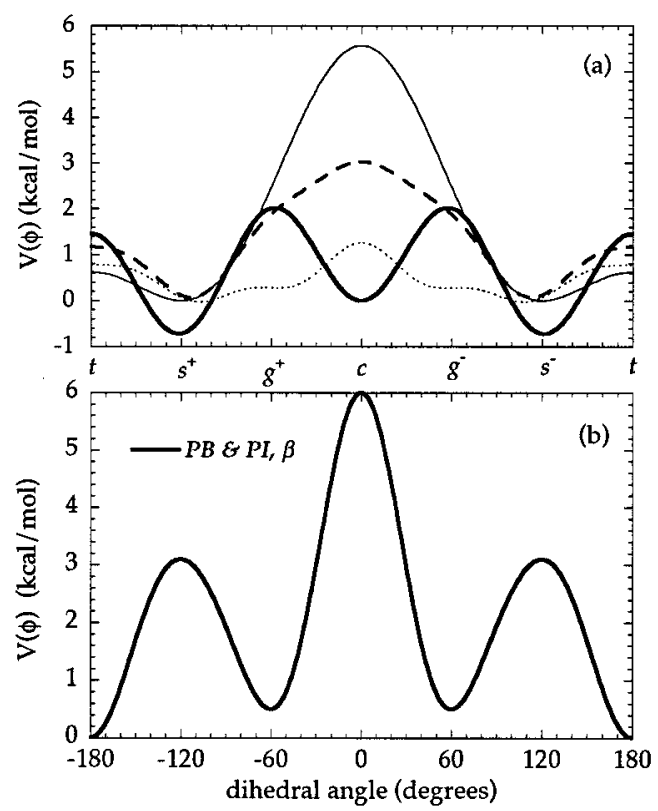

Figure 2. (a) Rotational potentials for the $\alpha_{1}$ bond (dotted curve) and $\alpha_{2}$ bond (thin solid curve) of cis-PI20 and for the $\alpha$ bonds of cis-PB (heavy solid and dashed curves). The dashed curve for the rotational potential of cis-PB $\alpha$ bonds is estimated from the MD simulation results of $\mathrm{Li}$ and Mattice, ${ }^{19}$ and the other curves are estimated from the simulations of Rey et al. ${ }^{20}$ The corresponding functional forms and energy parameters are listed in Table 1. (b) Rotational potentials for the $\beta$ bonds of cis-PI and cis-PB. See Figure 1 for the definition of bonds of different types.

ular dynamics (MD) simulations were presented in our earlier studies. ${ }^{3-9}$ In the following, we will present the results for cis-PI. Those for cis-PB will also be repeated and shown whenever necessary for comparative purposes.

Geometry and Conformational Energetics of cisPI in Comparison to cis-PB. The repeat units of cisPB and cis-PI are shown in Figure 1. PB comprises three types of bonds: two $\alpha$ bonds adjoining the double bond, one $\beta$ bond between two tetrahedrally bonded carbons, and a double bond, as shown in the figure. In PI, there are two distinct $\alpha$ bonds, due to the presence of the methyl side group. These will be designated as $\alpha_{1}$ and $\alpha_{2}$, the latter flanking the methyl-substituted backbone carbon.

In Figure 2a, the rotational potentials for the different bonds in cis-PB and cis-PI are shown. The dotted and thin solid curves refer to the rotational potentials of the $\alpha_{1}$ and $\alpha_{2}$ bonds of cis-PI, respectively. The dashed and heavy solid curves represent the potentials for the $\alpha$ bond of cis-PB, estimated from two different references, $\mathrm{Li}$ and Mattice ${ }^{19}$ and Rey et al., ${ }^{20}$ respectively. The potentials of $\mathrm{Li}$ and Mattice refer to the atom-based molecular dynamics (MD) simulation results for bulk cis-PB, in contrast to the Monte Carlo (MC) results of Rey et al. The former exhibits two minima, and the latter three; these will be accordingly referred to as the 
Table 1. Energy Parameters Used in Bond Rotational Potentials ${ }^{\mathbf{a}}$

\begin{tabular}{lccccccc}
\hline chain & bond & \multicolumn{1}{c}{$\mathrm{a}_{0}$} & \multicolumn{1}{c}{$\mathrm{a}_{1}$} & \multicolumn{1}{c}{$\mathrm{a}_{2}$} & \multicolumn{1}{c}{$\mathrm{a}_{3}$} & \multicolumn{1}{c}{$\mathrm{a}_{4}$} & \multicolumn{1}{c}{$\mathrm{a}_{5}$} \\
\hline cis-PB $^{\mathrm{b}}$ & $\alpha$ & 0.32 & -2.01 & -0.06 & 2.39 & 0 & 0 \\
cis-PB $^{\mathrm{c}}$ & $\alpha$ & 0.32 & -1.25 & 0.74 & 1.67 & 0 & -0.89 \\
cis-PI $^{\mathrm{b}}$ & $\alpha$ & 0.06 & -0.37 & 0.01 & 1.30 & 0.47 & -1.05 \\
cis-PI $_{1}$ & $\alpha_{2}$ & -1.28 & 1.28 & 0 & 0 & 0 & 0.30 \\
cis-PB $^{\mathrm{b}}$ & $\beta$ & 1.00 & 1.92 & -0.55 & -1.91 & 1.10 & -1.56 \\
cis-PIb $^{\mathrm{s}}$ & $\beta$ & 1.00 & 1.92 & -0.55 & -1.91 & 1.10 & -1.56
\end{tabular}

a Rotational potential: $\mathrm{V}=\mathrm{k}_{\phi} \sum \mathrm{a}_{\mathrm{i}} \cos \phi_{\mathrm{i}}$, where $0 \leq \mathrm{i} \leq 5$ and $\mathrm{k}_{\phi}$ $=1.934 \mathrm{kcal} / \mathrm{mol}$. ${ }^{\mathrm{b}}$ Using ref $20{ }^{\mathrm{c}}$ Using ref 19 .

2-state and 3-state rotational potentials. In the former, the isomeric states $\mathrm{skew}^{+}\left(\mathrm{s}^{+}\right)$and $\mathrm{skew}^{-}\left(\mathrm{s}^{-}\right)$are observed, with a relatively high barrier at the cis (c) state, practically preventing the rotational isomerization $\mathrm{S}^{+} \leftrightarrow \mathrm{S}^{-}$over the cis state. The transitions over the trans (t) state, on the other hand, are subject to low ( $\leq 2 \mathrm{kcal} /$ mol) energy barriers for the two models. See Table 1 for the functional form and parameters of the torsional potentials used in the present study.

In cis-PI, the bond $\alpha_{1}$ rotates almost freely, as may be deduced from the rotational potential displayed by the dashed curve in Figure $2 a$, whereas the rotations of bond $\alpha_{2}$ are more severely hindered. The latter exhibits two isomeric states, $\mathrm{s}^{+}$and $\mathrm{s}^{-}$, separated by a high barrier at position c, similar to the behavior of the $\alpha$ bonds of cis-PB observed in MD simulations. Thus, there is practically one accessible isomeric passage, $\mathrm{s}^{+}$ $\leftrightarrow \mathrm{s}^{-}$, across the trans state for the $\alpha_{2}$ bond, the transition across the cis state being highly improbable.

In contrast to the $\alpha$ bonds, the $\beta$ bonds exhibit three minima in both cis-PB and cis-PI, at positions t, gauche ${ }^{+}$ $\left(\mathrm{g}^{+}\right)$, and gauche $\mathrm{e}^{-}\left(\mathrm{g}^{-}\right)$. The passage between the gauche states over the cis state is practically impossible due to the high ( $=5 \mathrm{kcal} / \mathrm{mol}$ ) energy barrier, as may be seen in Figure $2 \mathrm{~b}$. Transitions between states $\mathrm{g}^{ \pm}$and $\mathrm{t}$ across the ${ }^{ \pm}$states also require relatively high energy barriers, compared to those involved in the accessible transitions ( $\mathrm{s}^{+} \leftrightarrow \mathrm{S}^{-}$across the t state) of $\alpha$ bonds. Therefore the $\alpha$ bond rotations are more likely to control the conformational relaxation of cis-PB and cis-PI.

In addition to these short-range conformational potentials, long-range interactions were taken into consideration in our calculations. To this end, all interatomic distances were checked throughout the time evolution of each conformational change. The conformational transitions involving interatomic contacts closer than the cutoff distance of $3.8 \AA$ were not accepted. This approach severely suppresses the passage over the cis state of the $\alpha$ bonds in either cis-PI or cis$\mathrm{PB}$, thus eliminating the qualitative differences that may arise from the adoption of the two different functions, 2-state and 3-state, for the torsional potential of $\alpha$ bonds.

Previous calculations for cis-PB were done, ${ }^{8}$ using the potential set given by Rey et al.$^{20} \mathrm{Good}$ agreement was obtained therein ${ }^{7}$ with experiments and previous ${ }^{17,18}$ MD simulations. Calculations have been repeated here with the potentials of $\mathrm{Li}$ and Mattice. The results concerning the mechanism of motion do not differ substantially from those based on the potentials of Rey et al. because the excluded-volume effect effectively hinders the access to the cis state of the $\alpha$ bond, which is the major difference between the two potentials. It is worth noting that, in the MD simulations of bulk cis$\mathrm{PB}$, the two equilibrium states $\mathrm{s}^{+}$and $\mathrm{s}^{-}$for the methine-methylene bonds were observed (in the dis- tributions of dihedral angles extracted from the MD trajectories) despite the implementation of a 3-fold potential exhibiting minima at states $\mathrm{C}, \mathrm{s}^{+}$, and $\mathrm{s}^{-}$. This is consistent with the fact that the cis state is not accessible in the present analysis when the excludedvolume effect or the second-order interactions between neighboring units along the backbone are taken into consideration.

Calculation Procedure. The chains are generated using the statistical weight matrixes of the rotational isomeric states (RIS) formalism given by Abe et al. ${ }^{23}$ Conformational potential energy curves are derived from statistical weight matrixes and from the work of Rey et al. ${ }^{20}$ The double bond is kept fixed at the cis state in our calculations. In view of the dependence of the results on the starting conformation, calculations are repeated for $500 \mathrm{M}$ onte Carlo chains, each of 25 bonds, generated by using the RIS scheme. The results refer to the averages over this ensemble of chains. In the CK model, the effects of frictional resistance and internal rotational barriers are expressed by the ratio

$$
\mathrm{k}_{0}=\left(2 \mathrm{k}_{\phi} \Delta \mathrm{t}\right) /\left(\mathrm{I}^{2} \xi\right)
$$

where I is the bond length taken as $1.53 \AA, \xi$ is the friction coefficient taken as $0.5248 \times 10^{-7} \mathrm{dyn} \cdot \mathrm{s} / \mathrm{cm},{ }^{10}$ $\mathrm{k}_{\phi}$ is the torsional energy amplitude which is equal to $1.93 \mathrm{kcal} / \mathrm{mol}$, and $\Delta \mathrm{t}$ is a parameter related to the time scale of the small steps used in calculations, taken as $10 \mathrm{ps}$, in conformity with our previous work.7,8 Substituting these values into eq 3 leads to $\mathrm{k}_{0}=0.22$.

\section{Results}

Mechanism of Conformational Relaxation. (a) $\alpha_{1}$-Bond Rotations. The mechanism of motion accompanying the rotational jump of $\alpha_{1}$ bonds in cis-PI is investigated first. In parallel with our earlier treatments, ${ }^{4,8}$ the mechanism is described in terms of (i) the most probable torsional motion of bonds around the $\alpha_{1}$ bond which undergoes a rotational isomerization, (ii) the average spatial reorientation of the neighboring backbone bonds, and (iii) the most probable displacements of atoms along the chain, after the completion of the isomeric jump. All these motions are generally confined to the nearest monomers flanking the rotating bond, except for the atomic displacements which are spread over a slightly longer distance al ong the chain.

The results are illustrated in Figure 3 . The rotating bond is assigned the index " 0 " al ong the abscissa. The responses of the remaining bonds along the chain are presented in the ordinate. Parts $a$ and $b$ exhibit the dihedral angle motions, part c shows the backbone $\mathrm{C}-\mathrm{C}$ bond reorientations, and part $\mathrm{d}$ shows the atomic displacements. For comparative purposes, the results obtained with cis-PB under the same environmental conditions are displayed by the dotted curves. Interestingly, the backbone motions in the two chains are very similar, despite the presence of the methyl side group in cis-PI. The only discernible features are the larger displacement of backbone atoms (part d) and the larger amplitude librational motions (part a) in cis-PI compared to those in cis-PB. Such slightly larger size cooperative motions in $\mathrm{PI}$ are understandable in view of the presence of the methyl side group, which should accommodate the conformational change. As pointed out above, although the conformational potential energy curve of the $\alpha_{1}$ bond is al most flat, the $\mathrm{s}^{+} \leftrightarrow$ ctransition 

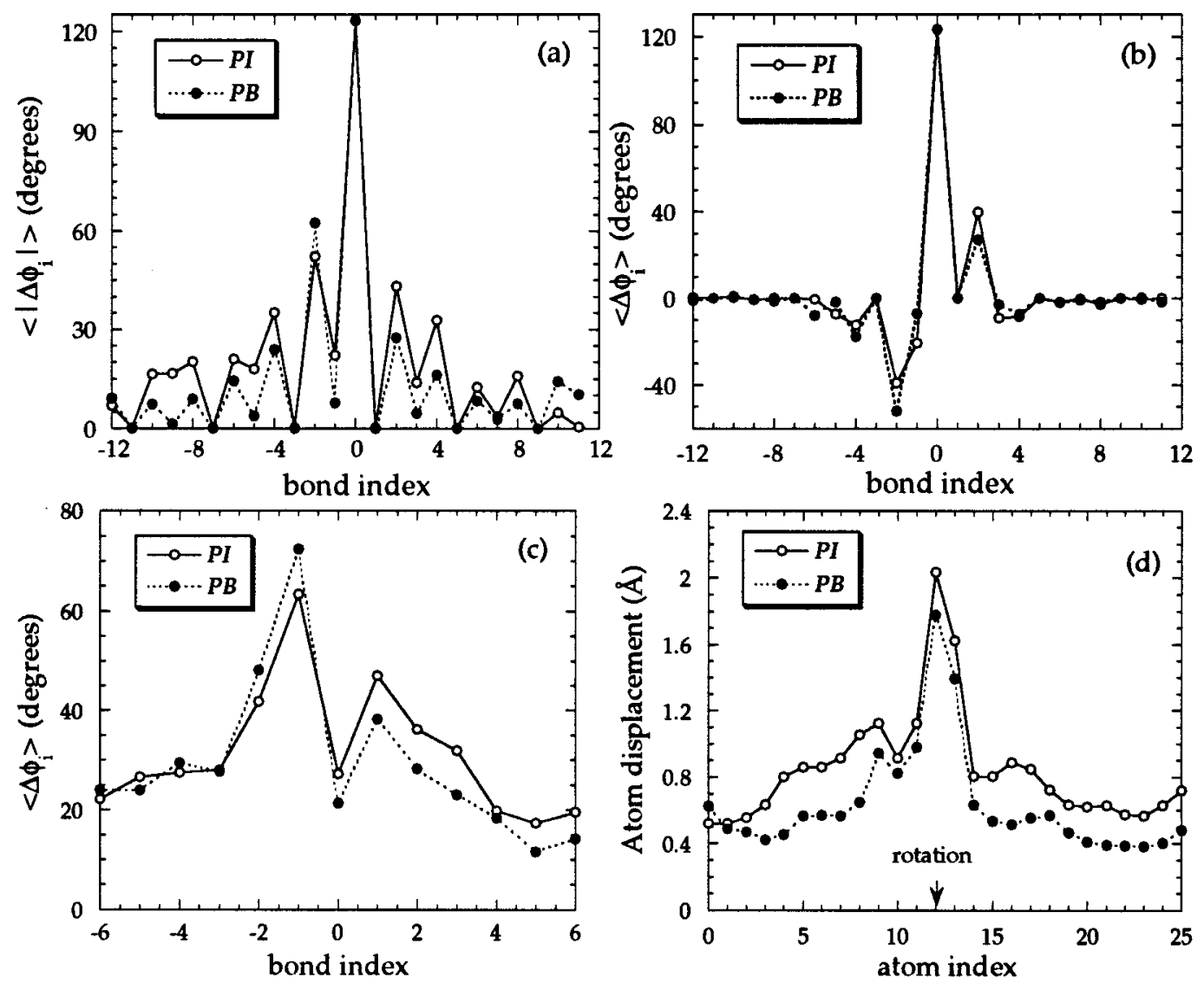

Figure 3. (a) Average absol ute changes in the dihedral angles of bonds, (b) average changes in the dihedral angles of bonds, (c) average spatial reorientation of bond vectors, and (d) average spatial displacements of atoms, in response to the rotation of $\alpha_{1}$ bonds of cis-PB and cis-PI.

is difficult because of the excluded-volume effect. Only $5 \%$ of all accessible transitions about the $\alpha_{1}$ bond are of the type $\mathrm{s}^{+} \leftrightarrow \mathrm{c}$, when the 3-state potential of Rey et al. is used in calculations.

(b) $\boldsymbol{\beta}$-Bond Rotations. Insofar as the motion of $\beta$ bonds is concerned, the backbone response is again al most identical in the two chains. Results of calculations for $\beta$-bond transitions are illustrated in Figure 4. The displacements of the backbone atoms of cis-PI are generally higher than their counterparts in cis-PB. This difference, observed despite the adoption of a common friction coefficient for the two chains, is indicative of the tendency of cis-PI chains to undergo larger size movements to accommodate bond isomerizations. The larger atomic displacements contribute to a larger work requirement for conformational relaxation in cis-PI compared to cis-PB and will be reflected in the correlation time (see eq 1 ) for the relaxation probed by NMR.

(c) $\alpha_{2}$-Bond Rotations. The only possible type of transition of the $\alpha_{2}$ bonds is that of the type $\mathrm{s}^{-} \leftrightarrow \mathrm{s}^{+}$ across thet state. The response of the chain to this type of transition was found to be qualitatively similar to that accompanying the same rotational jump of bond $\alpha_{1}$, further confirming that the mechanism of cooperative conformational relaxation driven by an isomeric jump is practically independent of the presence of the side group. The only net effects due to the side group are (i) an increase in atomic displacements evidently related to the accommodation of the side group and (ii) the lower frequency of occurrence of isomeric jumps, the access to some conformational passages being prevented by steric effects.
Correlation Times. Comparison with NMR Data. We now proceed to the determination of the terms appearing in eq 1 for estimating the relative correlation times for $\mathrm{C}-\mathrm{H}$ bond reorientations in cis-PI and cis-PB in comparison to NMR data.

The present CK analysis suggests that the extent of reorientation of the methylene $\mathrm{C}-\mathrm{H}$ bonds appended to the backbone, which are observed in NMR relaxation experiments, will be of comparable sizes in cis-PI and cis-PB. This is confirmed in Figure 5, where the reorientations of the $\mathrm{C}-\mathrm{H}$ bond vectors in response to the isomerization of a central $\alpha_{1}$ bond are shown to obey similar distributions in cis-PI and cis-PB. We note that one of the two methine groups of cis-PB repeat units is replaced by a methyl-appended carbon in cis-PI, and for this particular group, the $\mathrm{C}-\mathrm{H}$ bond reorientation noted in the ordinate actually refers to the reorientation of the $\mathrm{C}-\mathrm{C}$ bond between the backbone and the side group.

As explained in our earlier work, ${ }^{4}$ the extent of reorientation of $\mathrm{C}-\mathrm{H}$ bonds determines the front term $\tau_{0}$ in the rate expression presented in eq 1 . On the basis of the close similarity of the curves for cis-PB and cis$\mathrm{PI}$ displayed in Figure 5, we conclude that $\tau_{0}(\mathrm{cis}-\mathrm{PI}) \approx$ $\tau_{0}($ cis-PB $)$.

We now determine the relative contributions of the cumulative displacements and short-range conformational energies to $\tau_{\mathrm{c}}$ for cis-PI and cis-PB. A meaningful comparison of these terms requires us to adopt the same coefficient of friction for the two cases. The method of choice of the friction coefficients for the two polymers was discussed and their measurements were reported and discussed in detail by Lauprêtre et al. ${ }^{10} \mathrm{~F}$ rom these 

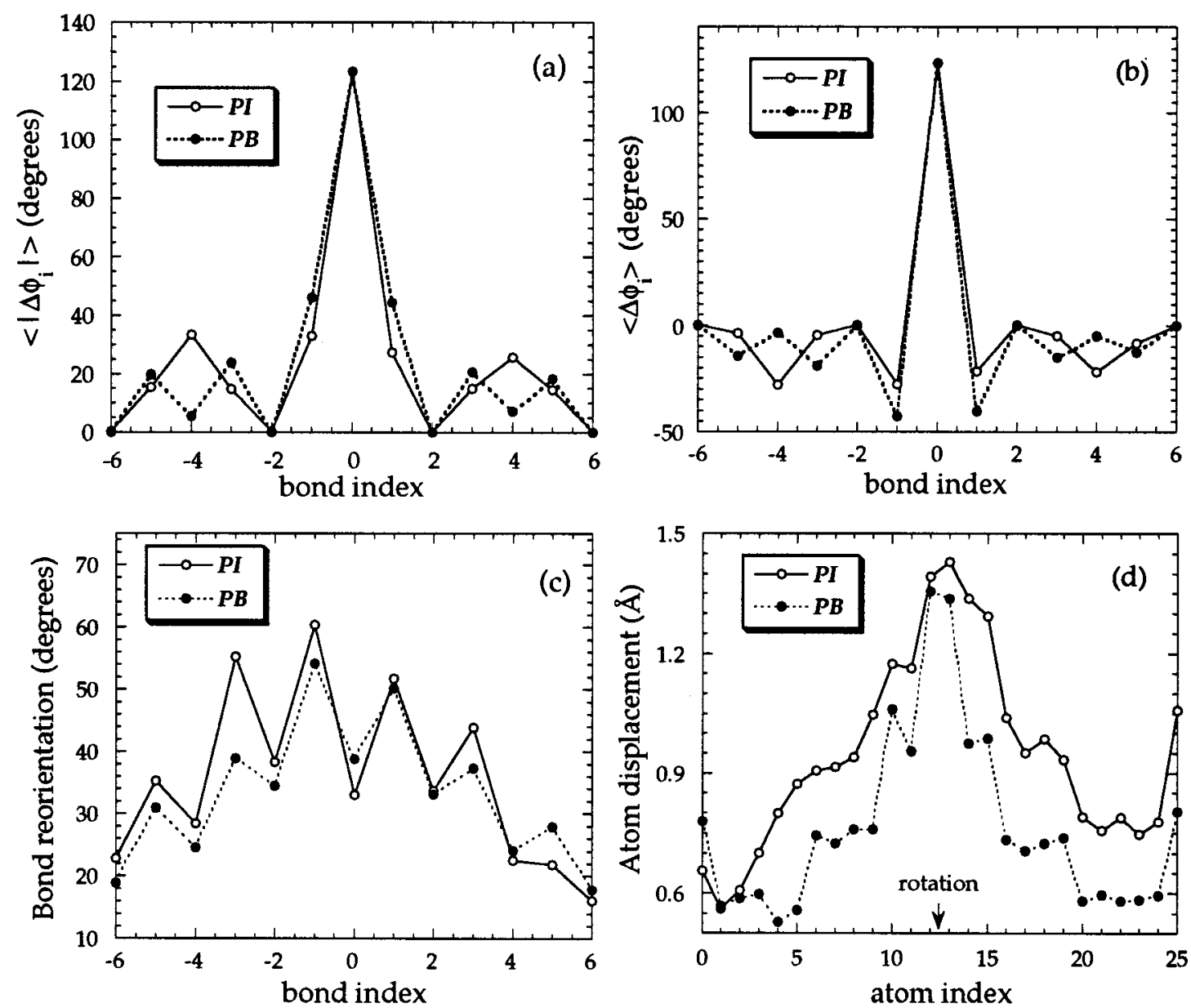

Figure 4. Response to the rotation of $\beta$ bonds of cis-PB and cis-PI. See caption for Figure 3.

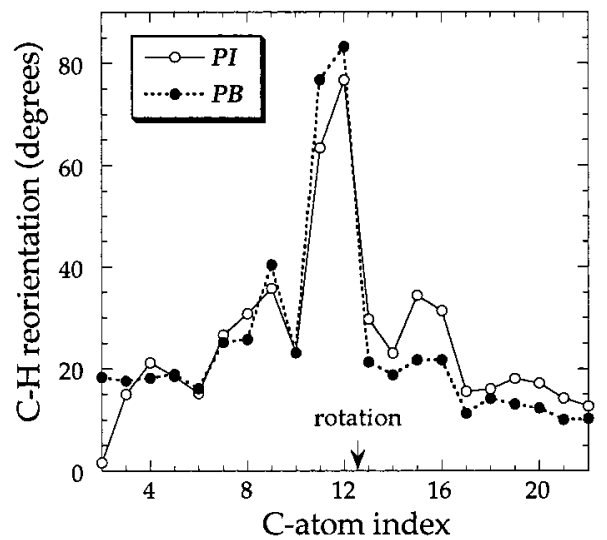

Figure 5. Reorientation of $\mathrm{C}-\mathrm{H}$ (or $\mathrm{C}-\mathrm{CH}_{3}$ ) bond vectors accompanying the isomerization of the $\alpha_{1}$ bond of cis-PB and cis-PI. The arrow shows the rotating bond.

data, the two polymers exhibit the same friction coefficient, $\xi=0.5248 \times 10^{-7} \mathrm{dyn} \cdot \mathrm{s} / \mathrm{cm}$ at $341 \mathrm{~K}$ for cis-PI and at $313 \mathrm{~K}$ for cis-PB. In Figure 6 the cumulative atomic displacements $\sum \delta \mathbf{R}_{\mathrm{i}} \cdot \delta \mathbf{R}_{\mathrm{i}}$ for cis-PI and cis-PB are compared for this value of the friction coefficient. The results are shown for $120^{\circ}$ rotations of $\alpha_{1}$ and $\alpha_{2}$ bonds of cis-PI and for the $\alpha$ bonds of cis-PB. $\beta$-Bond rotations are not included because of their low probability of occurrence relative to $\alpha$-bond rotations. The difference between cis-PI and cis-PB is predominantly due to the extra accommodations for the methyl group. This leads to a larger volume swept by the atoms and a larger work done against friction.

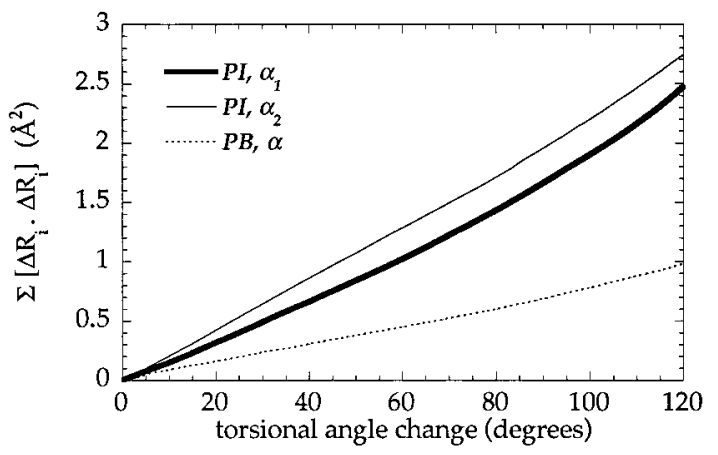

Figure 6. Cumulative square displacements of atoms during the isomerization of a central $\alpha$ bond in cis-PB and $\alpha_{1}$ and $\alpha_{2}$ bonds in cis-PI.

Inasmuch as the cumulative displacement is a factor directly affecting the observed correlation times, a careful analysis was performed for investigating its dependence on the functional form of the $\alpha$-bond rotation potential. Calculations were repeated for the 2-state and 3-state rotational potentials displayed in Figure 2 as the long-dashed and heavy-solid curves. The former 20 lead to $\sum \delta \mathbf{R}_{\mathrm{i}} \cdot \delta \mathbf{R}_{\mathrm{i}}=0.98 \AA^{2}$, for $\Delta \phi=120^{\circ}$, whereas the latter ${ }^{19}$ yiel d values in the range $\sum \delta \mathbf{R}_{\mathrm{i}} \cdot \delta \mathbf{R}_{\mathrm{i}}=0.58-1.48$ $\AA^{2}$ depending on the absolute value of the rotational barrier (see above). In view of the respective values 2.48 and $2.75 \AA^{2}$ obtained for cumulative square displacements accompanying the rotations of the $\alpha_{1}$ and $\alpha_{2}$ bonds of cis-PI, we conclude that the cumulative displacements $\left(\sum \delta \mathbf{R}_{\mathrm{i}} \cdot \delta \mathbf{R}_{\mathrm{i}} \approx 1 \AA^{2}\right)$ in cis-PB are about $2-3$ times smaller than those occurring in cis-PI. 

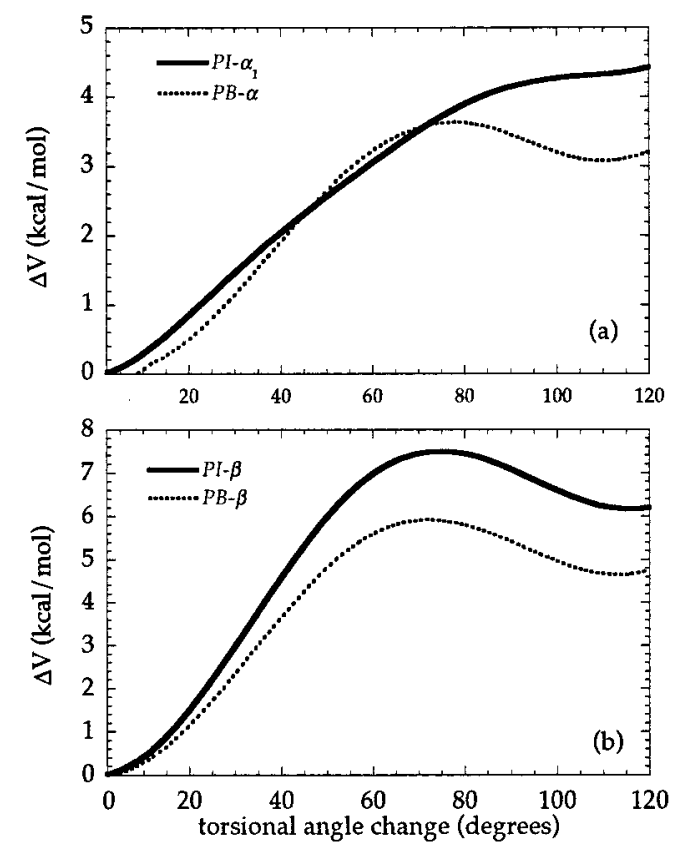

Figure 7. Conformational potential energy changes during the isomerization of (a) $\alpha$ and $\alpha_{1}$ bonds and (b) $\beta$ bonds of cis$\mathrm{PB}$ and cis-PI.

Finally we considered the contribution of rotational potentials to observed $\tau_{\mathrm{c}}$. This may be assessed by comparing the respective values of $\Delta \mathrm{V}$ obtained for cis$\mathrm{PB}$ and cis-PI bond rotations shown in Figure 7. $\Delta \mathrm{V}$ differs from the torsional potentials of the individual bonds displayed in Figure 2 because it is the effective change in internal energy for the overall chain resulting from the conformational rearrangements driven by a bond rotational jump. It can be seen that the value $\Delta \mathrm{V}$ required for an $\alpha_{1}$-bond rotation in cis-PI is higher than that of an $\alpha$ bond of cis-PB by about $1.0 \mathrm{kcal} / \mathrm{mol}$. The results for $\mathrm{PB}$ shown in Figure 7 are obtained with the 3 -state potential ${ }^{20}$ for the rotation of $\alpha$ bonds. Adoption of the 2-state potential ${ }^{19}$ for $\alpha$-bond rotation also yiel ds comparable results. The $\Delta \mathrm{V}$ changes involved in $\beta$-bond rotations of cis-PB and of cis-PI are shown in Figure 7b. The relatively higher energy barrier of cis-PI (by 1.5 $\mathrm{kcal} / \mathrm{mol}$ ) again leads to a longer correlation time for relaxation of cis-PI chains.

In summary, (i) the cumulative square displacements of atoms localizing and/or accommodating a bond isomerization differ in cis-PI and cis-PB by a factor of 2, the movements of cis-PI being larger in size and thereby involving longer correlation times according to eq 1, (ii) the short-range conformational energy accompanying isomeric transitions is higher by about $1 \mathrm{kcal} / \mathrm{mol}$ in cis$\mathrm{PI}$ compared to cis-PB, which leads to an effective ratio of $\exp [\Delta(\Delta \mathrm{V}) / \mathrm{RT}] \approx 5$ contributing to $\tau_{\mathrm{c}}(\mathrm{PI}) / \tau_{\mathrm{c}}(\mathrm{PB})$, and (iii) the mechanisms of backbone motion are closely similar in the two polymers, such that the front factors $\tau_{0}$, which reflect the amplitude of angular reorientations of the examined internuclear vectors, are almost identical for the two polymers. In view of these results, the observed correlation times for cis-PI are expected to be longer than those for cis-PB by about 1 order of magnitude. NMR measurements by Lauprêtre et al.10,12 indicate that the overall correlation time ratio of cis-PI to cis-PB is approximately 10 , in conformity with present theoretical estimates.

Conformational Relaxation Motions of cis-PI and cis-PB. For a more detailed understanding of the

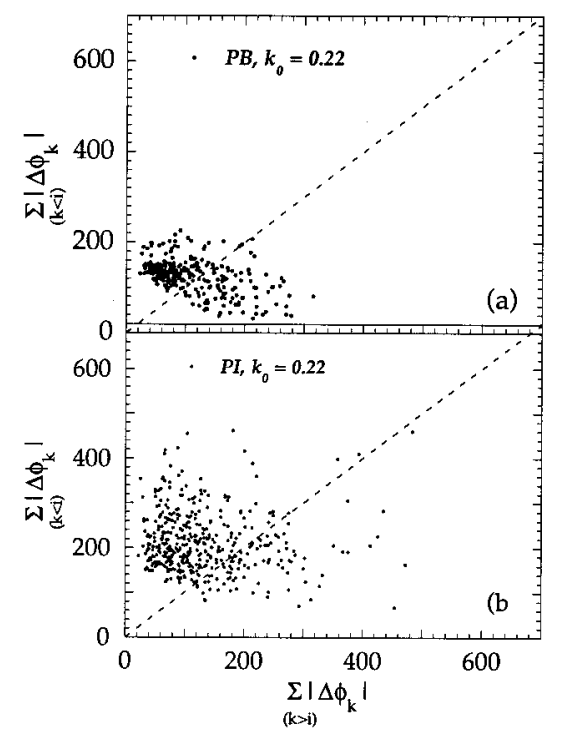

Figure 8. Overall response of all bonds located on the left and on the right of a rotating $\alpha_{1}$ bond, for cis-PB and cis-PI, in terms of absolute changes in dihedral angles for the subsets of motions which involve a barrier crossing.

mechanism of local dynamics in cis-PI in comparison to that in cis-PB, the following analysis was performed. The response to rotational transition was examined in a series of chains having different starting configurations. Precisely, the total sizes of torsional motions on both sides of the rotating bond were evaluated for each starting configuration. Results for an $\alpha_{1}$-bond rotation are displayed by the 2-dimensional plots presented in Figure 8. The ordinate and abscissa represent the summations $\sum\left|\Delta \phi_{\mathrm{k}}\right|$ over the absolute changes in dihedral angles of all bonds $\mathrm{k}>\mathrm{i}$ and $\mathrm{k}<\mathrm{i}$, respectively, when bond i completes a transition from one isomeric state to another.

For a symmetric chain, both sides of the diagonal should be equally populated, whereas in asymmetric chains such as PB or PI, a stronger response may be observed on one side of the rotating bond or the other, as may be seen in Figure 8.

The most striking difference between cis-PI and cis$P B$ is the broader dispersion of dihedral angle changes in the former, revealing that in cis-PI the conformational relaxation processes involve larger, but compensating, changes in dihedral angles, in general. Interestingly, cumulative dihedral angle changes up to $650^{\circ}$ may be observed on one side of cis-PI for particular conformations.

A similar analysis for the investigation of the mechanism of the local dynamics was performed with cumulative displacements of backbone atoms, $\sum\left|\delta \mathbf{R}_{\mathrm{k}}\right|$. The results are displayed in Figure 9.

Results presented in Figure 9 for two friction coefficients, varying by 1 order of magnitude, show that with increasing friction coefficients (smaller $\mathrm{k}_{0}$ ) smaller displacements are obtained in general. This is consistent with our previous analysis of the relative contributions of librations and coupled jumps in response to bond isomerization. ${ }^{8}$ The probability of occurrence of coupled jumps was shown therein to increase with increasing friction, because a more efficient localization of the motion is achieved by such cooperative rearrangements, as opposed to librations spread over long distances al ong the chain contour. The localization of the motion with 

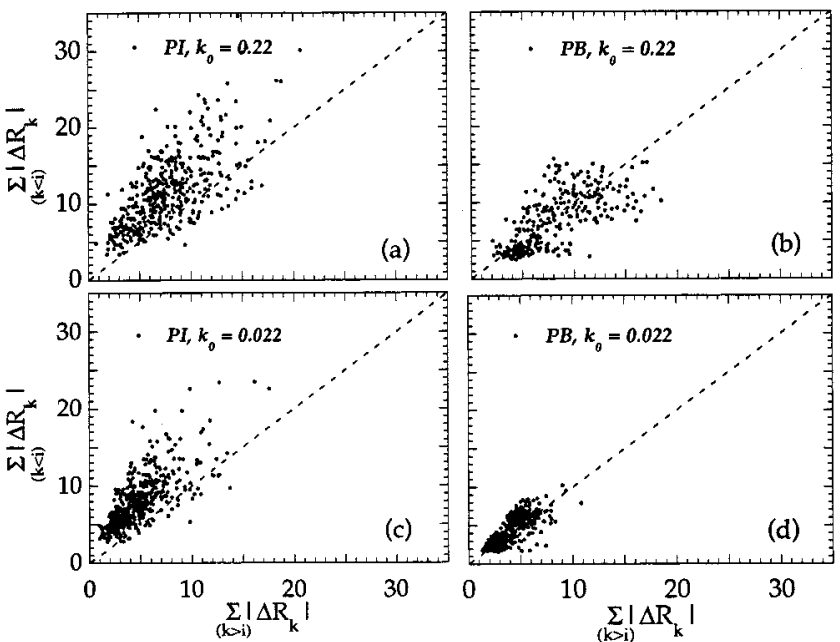

Figure 9. Overall response of all atoms located on the left and on the right of the rotating $\alpha_{1}$ bond of cis-PB and cis-PI in terms of atomic displacements. Subsets of motions which involve a barrier crossing are considered. Results are presented for $\mathrm{k}_{0}=0.22$ and $\mathrm{k}_{0}=0.022$. The lower value refers to a 10 fold increase in the effective friction coefficient.

increasing friction coefficient is clearly seen upon comparison of part a (or b) with part c (or d).

\section{Discussion and Conclusion}

The above analysis demonstrates that in dense media the mechanisms of backbone relaxational motions of cisPI and cis-PB are closely similar. In fact, the conformational rearrangements in response to a bond isomerization are shown to be almost identical in the two chains, apart from a general enhancement in the atomic displacements and/or dihedral angle motions in cis-PI compared to cis-PB. This increase in the size of local motions may be readily attributed to two factors: the need to accommodate the methyl side groups of cis-PI during conformational rearrangements and the reduced backbone flexibility of cis-PI arising from the fact that the cis state is practically inaccessible to PI bonds.

Despite the close similarity of backbone motions, two fundamental differences lead to a significant slowing down of the local motions in cis-PI compared to cis-PB for an identical value of the friction coefficient. The former is the need to accommodate the methyl side group during the conformational rearrangements of the backbone. The presence of the methyl group also hinders a number of transitions between isomeric states due to the excluded-volume effect occurring at the transition state between the initial and final conformations. This feature evidently leads to a larger vol ume swept by the moving atoms and, thereby, to a stronger resistance to motion, which is in turn reflected in the observed correlation times for cis-PI. Second, the short-range conformational energy requirements of local relaxation processes differ in the two chains. These factors lead to a ratio of about 10 for the correlation time of cis-PI to that of cis-PB, in accordance with the results from NMR relaxation experiments.

The mechanism of motion for cis-PB relaxation, presently obtained with the 2-state rotational potentials of $\mathrm{Li}$ and Mattice, ${ }^{19}$ is almost identical to that previously found ${ }^{4,7}$ with the 3 -state potentials of Rey et al. ${ }^{20}$ because the additional equilibrium state (cis) all owable in the latter is hindered in practice by the excludedvolume effect which is taken into consideration in the calculations. In fact, a lower bound, e.g. $3.8 \AA$, was implemented for the closest distance of approach of two sites (methylene, methine, or methyl groups) and transitions violating this excluded-vol ume requirement were rejected. As also pointed out in our previous studies, ${ }^{7}$ CK results for the mechanism of relaxation of cis-PB, displayed in Figures 3 and 4, are in good agreement with the results from the MD simulations of $\mathrm{Kim}$ and Mattice and of Gee and Boyd. ${ }^{18}$

Some similarities and differences between the results of the present study and previous MD simulations of cis-PI ${ }^{14-16}$ are as follows: Both approaches show that the cooperative motions that help to localize the conformational transitions are confined to a region of 1-2 repeat units. The same type of enhanced cooperativity of torsional motions of $\mathrm{PI}$ is predicted by both the present cooperative kinematics method and the MD simulations of Moe and Ediger. ${ }^{14}$ See, for example, Figure 5 in ref 14 in comparison to the present Figures $\mathrm{Bb}$ and $4 \mathrm{~b}$. Likewise, the $\mathrm{C}-\mathrm{H}$ bond reorientations displayed for $\alpha_{2}$-bond rotations in PI may be directly compared with the curve labeled "ab" in the Figure 8 of ref 16 . Therein, reorientations of $85-90^{\circ}$ were observed for the central two $\mathrm{C}-\mathrm{H}$ bonds, whereas our calculations indicate reorientations of about $75-80^{\circ}$ for the same bonds. The responses of the neighboring $\mathrm{C}-\mathrm{H}$ bonds differ, however, in the two studies. MD simulations yield a uniform reorientation angle of about $50^{\circ}$ for all neighboring bonds, whereas our calculations reveal a decrease with increasing separation from the rotating (central) bond. This difference may be attributed to the fact that the observed reorientations in MD simulations, uniformly spread over all neighbors, are driven by other transitions or librational motions occurring along the chain sequence, whereas the present CK results describe the response to a single-bond rotation, thus extracting information on the type of cooperative rearrangements accompanying an individual isomerization.

In the work of Moe and Ediger, the cooperativity between neighboring bond motions is emphasized to be mostly due to cooperative librations resulting from MD simulations, ${ }^{16}$ whereas a direct justification of this is not possible in the CK method. In fact, the descriptions of librations in the two approaches are somewhat different. In MD simulations, librations are obtained directly as a result of simulations, whereas in the CK calculations, the analytically determined most probable small-angle torsional rearrangements in the neighborhood of a given bond undergoing a rotameric transition are defined as librations.

The CK calculations show that a $120^{\circ}$ isomerization of an $\alpha_{2}$ bond requires a higher energy change compared to that for the $\alpha_{1}$ bonds. This is in qualitative agreement with the MD results ${ }^{15}$ where the conformational transition times of the two bonds are 97 and 63 ps, respectively. The predictions of the two methods for the transition times of the $\beta$ bonds are in disagreement, however. MD results indicate that the transition times of the $\alpha_{2}$ and $\beta$ bonds are comparable (97 vs 90 ps), whereas CK yields a higher effective energy for the isomerization of the $\beta$ bond (see parts a and b of Figure $7)$. This difference may arise from the lack of precise knowledge on the values of the rotational energy barriers between the isomeric states. The present higher mobility of the $\alpha_{2}$ bond can be understood from the examination of the rotational potential curves displayed in Figure 2. The thin-solid curve displayed for bond $\alpha_{2}$ 
in part a readily allows for a fast passage between states $\mathrm{s}^{+}$and $\mathrm{s}^{-}$across state $\mathrm{t}$, which may be responsible for the apparent faster transition rates of the $\alpha_{2}$ bonds in CK. An increase in this barrier may slow the apparent transition rates and yield transition times comparable to those of the $\beta$ bonds, as suggested by the comparable heights of the energy barriers in the cis state. Nevertheless, the extent of cooperativity between neighboring bonds along the cis-PI chains predicted by the present CK method is in general agreement with results of CK and MD calculations, as may be verified by comparison with results of Moe and Ediger. ${ }^{14-16}$

The CK method assumes the transition of a single bond from one isomeric state to another as the elementary event and calculates the effective changes in the chain energy or any other configurational parameter resulting from this elementary event. The choice of the elementary event starting and ending at a torsional energy minimum is appropriate as the MD calculations of Takeuchi and Roe on bulk polyethylene have shown. ${ }^{22}$ According to their calculations, at temperatures above $T_{g}$, each bond spends most of its time in one of the three isomeric minima. The frequency of transitions from one isomeric state to another is strictly governed by temperature, but the loci of the minima are unaffected by temperature.

It should be noted that the above approach provides at the same time (i) insights as to the molecular mechanism of local relaxational motions in particular polymers and (ii) a quantitative assessment of the relative relaxation rates (or relaxation times) of specific chains. The adoption of the same monomeric friction coefficient for different polymers by suitably selecting the temperature ${ }^{10}$ permits us to extract the difference in the correlation times of different polymers induced purely by the specific conformational geometry and energetics, exclusively.

Finally, a major advantage of the CK theory is its computational efficiency. CK results are found from the analytical solution of a series of coupled equations. Results in Figures 3-5, which would otherwise necessitate expansive, time-consuming MD simulations, are obtainable within computation times at least 2 orders of magnitude shorter than those of conventional MD simulations.

Acknowledgment. Helpful discussions with Prof. Turkan Haliloglu and partial support from the TUBITAK-CNRS Collaborative Research Grants Program and from the Bogazici University Research Fund (Project No. 98P001) are gratefully acknowledged.

\section{References and Notes}

(1) Baysal, C.; Atilgan, A. R.; Erman, B.; Bahar, I. J . Chem. Soc., Faraday Trans. 1995, 91, 2483.

(2) Baysal, C.; Atilgan, A. R.; Erman, B.; Bahar, I. Macromolecules 1996, 29, 2510.

(3) Bahar, I.; Erman, B.; Monnerie, L. Adv. Polym. Sci. 1994, $116,145$.

(4) Baysal, C.; Erman, B.; Bahar, I.; Lauprêtre, F.; Monnerie, L. Macromol ecules 1997, 30, 2058.

(5) Bahar, I.; Erman, B.; Monnerie, L. Macromol ecules 1992, 25, 6309, 6315.

(6) Bahar, I.; Baysal, N.; Erman, B.; Monnerie, L. Macromolecules 1995, 28, 1038.

(7) Baysal, C.; Bahar, I.; Erman, B.; Monnerie, L. Macromolecules 1996, 29, 2980.

(8) Haliloglu, T.; Bahar, I.; Erman, B.; Mattice, W. L. Macromolecules 1996, 29, 8942.

(9) Bahar, I. Macromol. Theory Simul. 1997, 6, 881.

(10) Lauprêtre, F.; Bokobza, L.; Monnerie, L. Polymer 1993, 34 , 468.

(11) Adolf, D. B.; Ediger, M. D. Macromolecules 1991, 24, 5834.

(12) Dejean de la Bâtie, R.; Lauprêtre, F.; Monnerie, L. Macromolecules 1989, 22, 122.

(13) English, A. D. Macromolecules 1985, 18, 178.

(14) Moe, N. E.; Ediger, M. D. Polymer 1996, 37, 1787.

(15) Moe, N. E.; Ediger, M. D. Macromolecules 1995, 28, 2329.

(16) Moe, N. E.; Ediger, M. D. Macromolecules 1996, 29, 5484.

(17) Kim, E.-G.; Misra, S.; Mattice, W. L. Macromolecules 1993, $26,3424$.

(18) Gee, R. H.; Boyd, R. H. J . Chem. Phys. 1994, 101, 8028.

(19) Li, Y.; Mattice, W. L. Macromolecules 1992, 25, 4942.

(20) Rey, A.; Kolinski, A.; Skolnick, J .; Levine, Y. K. J . Chem. Phys. 1992, 97, 1240.

(21) Kim, E.-G..; Mattice, W. L. J . Chem. Phys. 1994, 101, 6242.

(22) Takeuchi, H.; Roe, R. J . Chem. Phys. 1991, 94, 7446.

(23) Abe, Y.; Flory, P. J. Macromolecules 1971, 4, 230.

MA9811167 